\title{
Prediction of Shrinkage Defects in Iron Castings Using a Microporosity Model
}

\author{
Sung-Bin $\mathrm{Kim}^{1, \mathrm{a}^{*}}$, Young-Hoon Yim ${ }^{1}$, \\ Joong-Mook Yoon ${ }^{1}$, and Doru M. Stefanescu, ${ }^{2, b}$
} $\begin{aligned} &{ }^{1} \text { AnyCasting Software Co., Ltd., Woolim bldg. B-16 } \text { fl., } 583 \text { Yangcheon-ro, Gangseo-gu, } \\ & \text { Seoul, Korea } 07547\end{aligned}$

${ }^{2}$ Dept. of Materials Science \& Engineering, Ohio State University, 2041 College Rd Columbus, $\mathrm{OH} 43210$, USA

aksb@anycasting.com, ${ }^{\mathrm{b}}$ stefanescu.1@osu.edu

Keywords: microporosity formation, macro and micro shrinkage, nucleation of gas, cast iron

\begin{abstract}
A numerical model for prediction of shrinkage defects in iron castings has been developed. The model is based on gas pores evolution during solidification. It describes the evolution of gas concentration using mass conservation, and the change in melt pressure due to solidification contraction using Darcy's equation, with mixture continuity assumption in the liquid and the mushy zone. Gas pores nucleation has been calculated using the partial pressure of gas obtained from Sievert's law. The growth of porosity has been estimated using an equation based upon the total melt pressure on the pore, concentration and temperature of the gas. The porosity model was calibrated against literature data for microporosity, and then applied to the prediction of shrinkage defects in a ductile iron casting. Comparison between the model predictions and experimental measurements indicated that the porosity model can be applied not only to the prediction of micro-shrinkage but also to that of macro-shrinkage. Existing shrinkage prediction models based upon thermal models, such as Niyama criterion and the modulus of retained melt in mushy regions cannot predict correctly both micro- and macro-shrinkage.
\end{abstract}

\section{Introduction}

It is well established that high microporosity can result in dramatic deterioration of the mechanical properties, and in particular of fatigue properties. Because of the complexity of the physics associated with microporosity formation, assessing the hierarchy of the process and material parameters that affect microporosity is not a trivial task. A large number of computational models attempting to describe porosity occurrence is available in the technical literature (see for example early papers by Piwonka and Flemings [1] or Kubo and Pehlke [2], and the reviews in ref. [3,4]). The model proposed by Niyama et al. [5], which relates the occurrence of microporosity in steel castings to local thermal parameters such as the cooling rate and temperature gradient (the Niyama criterion) is relatively easy to implement in computational models. However, this criterion is only a qualitative indicator of shrinkage distribution. For quantitative prediction of shrinkage size, models must solve the heat transfer and phase change problem, and account for the liquid flow through the mushy zone and the nucleation and growth of gas bubbles $[6,7,8,9]$. More recently, a model that combines the Niyama criterion with the temporal evolution of the pore size was also proposed [10].

One approach consists in assuming that the growth of the gas pores in the mushy zone can be understood from the analysis of the local pressure [11]. However, the analysis must include nucleation and kinetic effects, which may delay pore formation predicted by thermodynamic equilibrium.

For aluminium alloys, there is significant experimental evidence that suggests that gas pores nucleate heterogeneously on inclusions that are present in the melt, e.g. ref. [12,13,14]. Another nucleation assumption stipulates nucleation on bifilms [15]. While the occurrence of bifilms favours nucleation of gas pores, it is not a condition sine qua non, as gas bubbling ensues in liquids supersaturated with gas when pressure is decreased (open a coke can for experimental proof). It is 
reasonable to conclude that nucleation of gas bubbles before the beginning of solidification occurs at the mould walls and on impurities in the liquid. Furthermore, micrometer-size gas bubbles can be assumed to exist in the liquid following their incorporation from the turbulent flow during pouring and mould filling. After the onset of solidification, the available solid phase could act as nucleation sites for the gas bubbles. Thus, bubble/pore nucleation is a hard to quantify, but decisive step in porosity formation in solidifying metals.

The metal pressure must be calculated by solving the governing fluid flow equations in the fully liquid regions (Navier-Stokes) and the mushy zone (Darcy's law). This can be a daunting task as the solution convergence requires large computational times. A simplified model for calculating the liquid alloy pressure during casting solidification was proposed by Catalina and Bates [16]. By making use of the continuity condition and taking advantage of specific conditions at the solid boundaries during the solidification process, the governing equations of flow were rewritten in a form that can be easily integrated for pressure. However, gas bubbling and pore growth were not considered. Porosity was assumed to occur only because of insufficient liquid feeding in the interdendritic channels.

\section{Review of Previous Works}

Previous work at AnyCasting [17] included the development of several criterion-based models: 1) a Niyama model, $\left.C_{\text {Niyama }}=G /(d T / d t)^{0.5} ; 2\right)$ a modified Niyama model, $\left.C_{N i y a m a}=G / t_{s} ; 3\right)$ a feeding efficiency model, $\left.C_{F / E f f}=(G / V)^{0.5} ; 4\right)$ a retained melt surface (RMS) and retained melt modulus (RMM) models. In these equations, $G$ is the temperature gradient, $T$ is the temperature, $t$ is the time, $t_{s}$ is the solidification time, and $V$ is the solidification velocity.

The RMM model calculates the retained melt modulus as $M_{S M}=V_{S M} / S_{S M}$, where $V_{S M}$ is the retained melt volume, and $S_{S M}$ is the retained melt surface. This model requires the selection of the critical solidification fraction (CSF), which is the solid fraction at which the dendritic network is significant. It depends on the solidification characteristics of the alloy. The solidification parameters required to predict shrinkage defects, such as $G, t_{s}, S_{S M}, V_{S M}$, etc., are calculated at CSF.

As discussed, criteria-based models can only attempt to predict shrinkage distribution, as exemplified in Fig. 1-a. To predict shrinkage size, a probabilistic model was developed [17]. Its principle is summarized in Fig. 2.

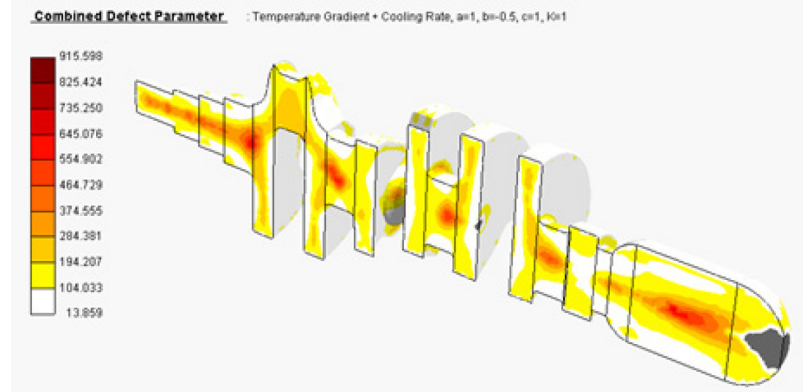

a) shrinkage distribution with the Niyama criterion

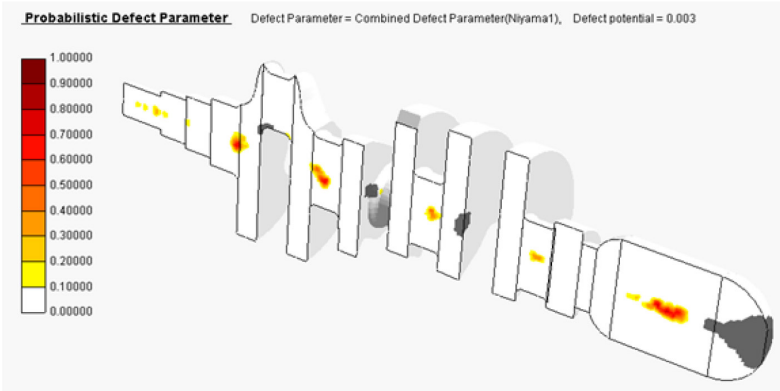

b) shrinkage size (volume) with the Niyama criterion combined with the probabilistic model

Fig. 1. Shrinkage predictions in a ductile iron crankshaft with Niyama criterion based models.

The total shrinkage volume of the casting, which depends on the type of alloy and the feeding capacity of the gating system, has been converted to the probability distribution function $\left(P_{s h}\right)$ and the area of its function as shown in Fig. 2. The shrinkage probability is reduced by the feeding capacity of the gating system. The remaining shrinkage probability is indicated as shrinkage potential, $\beta$ '. The amount of shrinkage in a region smaller than $\beta$ ' is added to a region larger than $\beta$ ', and the size of the shrinkage can be predicted stochastically. In this process the amount of shrinkage volume change due to the selection of $\beta$ ' is preserved, so that shrinkage conservation can be maintained. 
An example of the combined use of the Niyama criterion model and the probabilistic model is shown Fig. 1-b. and of the RMM and probabilistic models in Fig. 2.

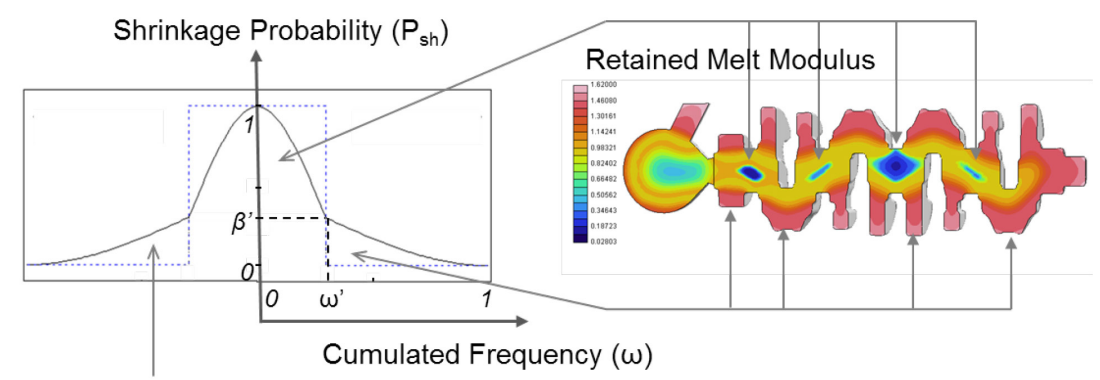

Shrinkage Probability :

$P_{S h}= \begin{cases}\beta^{\prime}+\left(1-\beta^{\prime}\right) \cos \left(\frac{\omega}{2 \beta^{\prime}} \pi\right) & \text { for } \quad \omega<\omega \\ \beta^{\prime}\left[1-\sin \left(\frac{\omega-\beta^{\prime}}{2\left(1-\beta^{\prime}\right)} \pi\right)\right] & \text { for } \quad \omega \geq \omega^{\prime}\end{cases}$

Shrinkage Conservation :

$\int_{0}^{1} P_{S h} d \omega=\beta^{\prime} \beta^{\prime}$ : Shrinkage Potential
Shrinkage Area Size
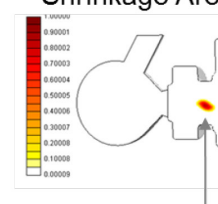
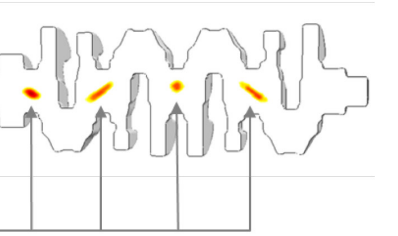

Fig. 2. Theory of probabilistic model combined with RMM for the calculation of shrinkage size (area) in a cast iron crankshaft.

\section{Physics of Model}

The principle of the shrinkage induced gas porosity (SIGAP) model is summarized in Fig. 3. It is based on the fact that the gas dissolved in the melt accumulates at the liquid/solid interface during solidification. As increased gas concentration produces higher partial pressure, gas bubbles are generated. The size of the bubble depends upon gas solubility, which in turn depends on temperature, pressure, composition, and solidification characteristics of the alloy. The gas bubbles generated after dendrite coherency (the value of solid fraction below which the Navier-Stokes equations are applicable) are trapped in the dendrite network, prevent feeding and generate porosity in the solid casting. For additional details on the physics of microporosity formation see Ref. [11].

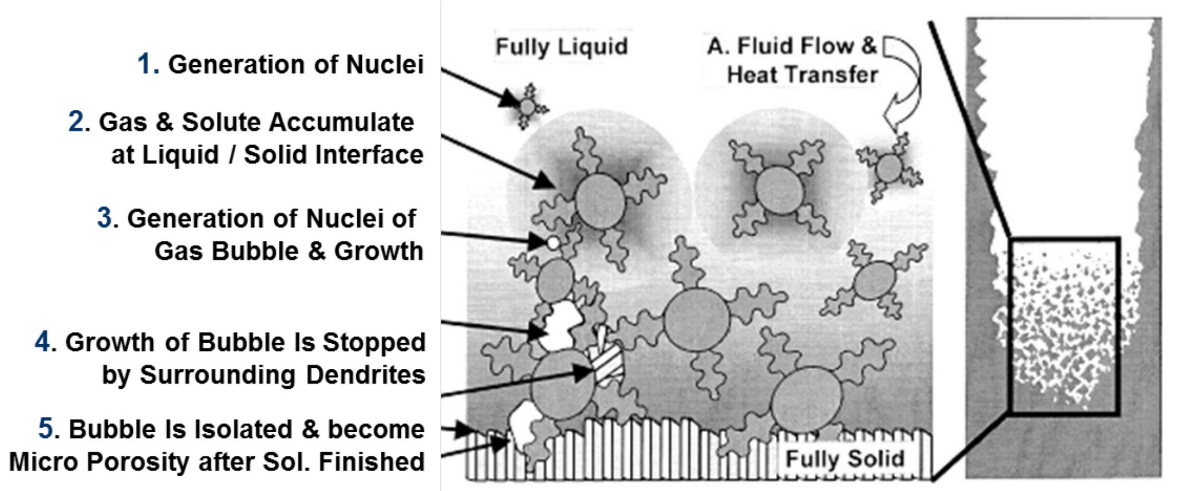

Fig. 3. Generation mechanism of microporosity in castings [3].

\section{Model Description}

The growth of the gas pores in the mushy zone can be understood from the analysis of the local pressure described by the following equation:

$$
P_{\text {tot }}=P_{f}+P_{s t}-P_{\text {shr }}+P_{\text {ext }} \text {. }
$$

where $P_{f}$ is the pressure resulting from melt flow, $P_{s t}$ is the metallostatic pressure, $P_{s h r}$ is the negative pressure from resistance to shrinkage induced flow through the fixed dendrite network, $P_{\text {ext }}$ is the pressure applied on the melt or partial squeezing. For a gas pore (bubble) to form in the liquid it needs to overcome, in addition to the total pressure, $P_{t o t}$, the capillary pressure. Note that this 
equation includes the additional term, $P_{f}$, compared to Eq. 2 in Ref [11], but does not include the graphite expansion pressure, which is not accounted for at this time.

Pressure in the melt. Once a third phase, pores, forms in the system, the mixture continuity equation gives:

$$
\frac{\partial\left(f_{s} \rho_{s}+f_{l} \rho_{l}+f_{p} \rho_{p}\right)}{\partial t}+\nabla \cdot\left(\rho_{l} \boldsymbol{V}_{l}\right)=0 .
$$

where the subscripts $s, l, p$ stand for solid, liquid and pores (gas), and $f$ is the fraction of phase. The velocity of the liquid, $\boldsymbol{V}_{\boldsymbol{l}}$, is calculated from Darcy's equation, as suggested by Kubo and Pehlke [2]:

$$
\boldsymbol{V}_{\boldsymbol{l}}=-\frac{K}{\mu_{l}}\left(\nabla P-\rho_{l} g\right) \text {. }
$$

where $K$ is the permeability of the mixture, and $\mu_{l}$ is the viscosity of the melt. Permeability is calculated as:

$$
K=\frac{\left(1-f_{s}-f_{p}\right)^{3} \lambda_{2}^{2}}{180\left(f_{s}+f_{p}\right)^{2} F_{k}}
$$

Here, $\lambda_{2}$ is the secondary arm spacing, and $F_{k}$ is a switching function for viscosity, introduced to smooth the transition from stage 1 (the solid phase moves with the liquid phase; relative velocity between solid and liquid is zero) to stage 2 (no solid movement after dendrite coherency) of solidification [18].

$$
F_{k}=0.5+\frac{1}{\pi} \tan ^{-1}\left[s\left(f_{s}-f_{c r}\right)\right] .
$$

where $s$ is a constant, and $f_{c r}$ is the critical solid fraction (constant), The secondary arm spacing can be calculated as a function of the local solidification time, $\Delta t_{f}^{n}$, or of the cooling rate.

$$
\lambda_{2}=a \Delta t_{f}^{n} \quad \text { or } \quad \lambda_{2}=a(\partial T / \partial t)^{n} .
$$

where $a$ is a coefficient obtained by fitting experimental data. The fraction of liquid is:

$$
f_{l}=1-f_{s}-f_{p} \text {. }
$$

When Eq. 3 and Eq. 7 are substituted into Eq. 2, a Poisson equation is obtained:

$$
\nabla\left(\frac{K}{\mu_{l}} \nabla P_{f}\right)=\left(\frac{\rho_{s}}{\rho_{l}}-1\right) \frac{\partial f_{s}}{\partial t}-\frac{\partial f_{p}}{\partial t}+\nabla \cdot\left(\frac{K}{\mu_{l}} \rho_{l} g\right) .
$$

The pressure in the melt, $P_{f}$, is obtained from this equation. It should be noted that for regular size casting the pressure in the melt is negligible compared to the metallostatic pressure. However, it becomes important for die castings.

Pore nucleation. It is assumed that nucleation occurs when the pressure in the pore exceeds the total pressure on the pore:

$$
P_{p} \geq P_{f}+P_{s t}+\frac{2 \sigma}{r}-P_{s h r}+P_{\text {ext }} .
$$

where $P_{p}$ is the pressure in the pore, $P_{f}$ is the melt pressure from Eq. $8,2 \sigma / r$ is the capillary pressure, $r$ is the pore radius, and $\sigma$ is the surface tension. The pore radius is taken as $r=0.5 \lambda_{2}$.

The pressure in the pore is calculated as:

$$
P_{p}=\left[100 C_{l}\left(\frac{A}{K_{e}}\right)\right]^{2} \times 101,325 \text { in }[P a] .
$$

where $A$ and $K_{e}$ are the activity and equilibrium coefficient of pores respectively, and 101,325 is a unit transformation factor. The equilibrium coefficient of pores is calculated as:

$$
\log \left(K_{e}\right)=-\frac{a}{T}-b \text {. }
$$

where $a, b$ are constants obtained through experimental fitting. 
The gas concentration in the liquid, $C_{l}$, is calculated from the mass conservation equation [19]:

$$
\frac{\partial\left(\rho C_{m}\right)}{\partial t}+\nabla \cdot\left(\rho \boldsymbol{V} C_{m}\right)=\nabla \cdot\left(\rho f_{l} D_{l} \nabla C_{m}\right)+\nabla \cdot\left(\rho f_{l} D_{l} \nabla\left(C_{l}-C_{m}\right)\right)-\nabla \cdot\left(\rho\left(\boldsymbol{V}-\boldsymbol{V}_{\boldsymbol{s}}\right)\left(C_{l}-C_{m}\right)\right) .
$$

where $\rho$ is the density of the solid/melt mixture, $C_{m}$ is the gas concentration in the solid/melt mixture, $\boldsymbol{V}$ is the velocity of the mixture, $\boldsymbol{V}_{\boldsymbol{s}}$ is the velocity of the solid, $f_{l}$ is the fraction of liquid, $D_{l}$ is the diffusivity of gas in the melt, and $C_{l}$ is the concentration of gas in the melt.

Growth of Porosity. The fraction of pores growing in the liquid and the mushy zone is calculated as:

$$
f_{p}=\alpha \frac{C_{l} T}{P_{t o t}} .
$$

where $\alpha$ is a transition factor from gas and melt mixture to pore, and $P_{t o t}$ is the total melt pressure on the pore calculated with Eq. 1. The transition factor is evaluated by comparing model predictions with experiments. An important feature of this model is that the gas concentration in the liquid is calculated at each time step through Eq. 12, which accounts for the redistribution of gas at the solid/liquid interface.

\section{Model Verification}

For the verification of SIGAP model, its predictions were compared with the experimental data from the literature [20] in which an unmodified A356 (Al-7Si-0.3Mg) alloy was directionally solidified in a tapered, cylindrical mold against a water-cooled chill, as shown in Fig. 4-a. The porosity measurements showing the variation with distance from the chill along the centerline, which were obtained by image analysis and data smoothing by $10 \mathrm{~mm}$ sampling area, are shown in Fig. 4-b. The porosity distributions of RMM and Niyama criterion combined with the probabilistic model, respectively, show the highest value near the top of casting, as seen in Fig. 4-c and -d, which is higher than the measured data shown in Fig. 4-b. However, the SIGAP model shows the highest value in the area marked as position 4 on Fig. 4-a and the distribution of porosity over the whole casting area, which is similar to the experimental results. 


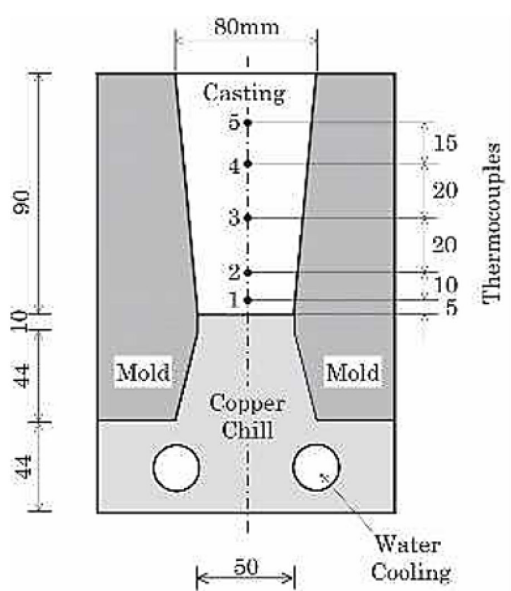

a) schematic illustration of experimental setup

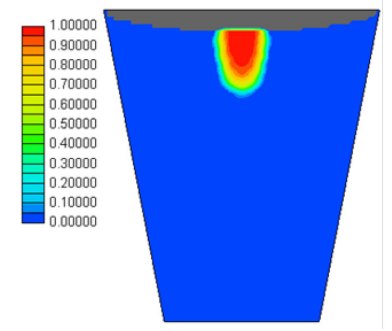

c) Retained melt modulus combined with the probabilistic model

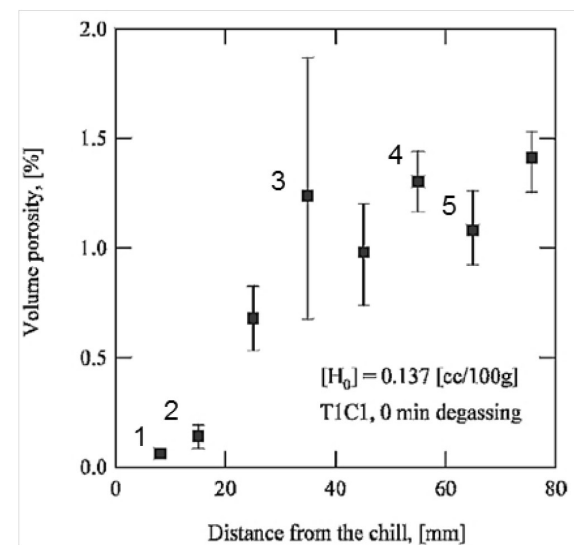

b) porosity measurement by image analysis and data smoothing by $10 \mathrm{~mm}$ sampling area

Fig. 4. Verification of the SIGAP model with experiment and comparison with criterion-based models.

Fig. 5-a presents the measured porosity distributions in casting with relatively low and high initial hydrogen content, of 0.1 and $0.137 \mathrm{cc} / 100 \mathrm{~g}$, respectively. The predicted values for 0.1 (triangles) and for $0.137 \mathrm{cc} / 100 \mathrm{~g}$ (squares), show good agreement with the experiments, except for the bottom area where the prediction is for very low gas porosity.

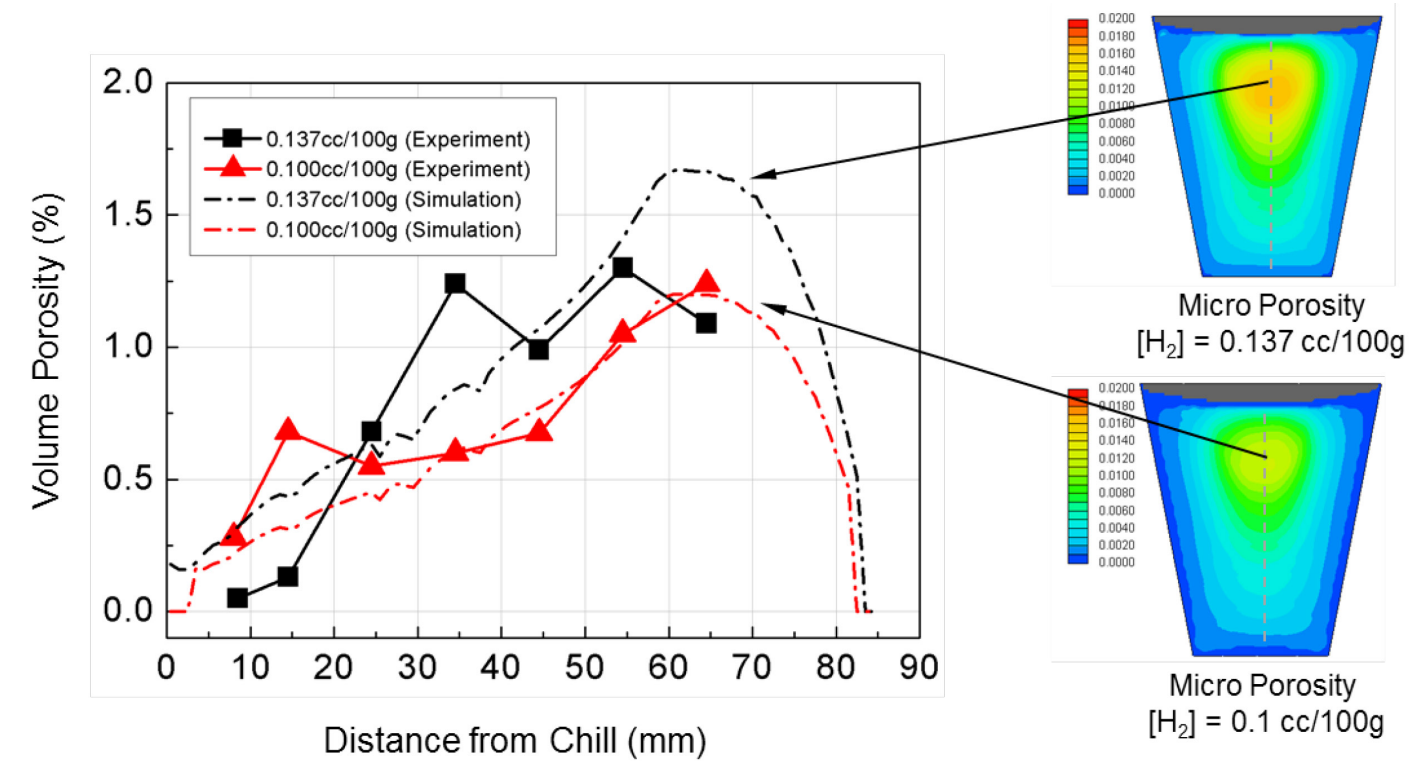

a) comparison SIGAP prediction with porosity

b) porosity distribution with measurement by image analysis $\left[\mathrm{H}_{2}\right]=0.1$ and $0.137 \mathrm{cc} / 100 \mathrm{~g}$

Fig. 5. Verification of SIGAP prediction with experiments with $\left[\mathrm{H}_{2}\right]=0.1$ and $0.137 \mathrm{cc} / 100 \mathrm{~g}$, respectively. 
The model predictions were evaluated for actual applications of ferrous alloy, such as diffuser castings made of ductile iron. A solid model of the casting and gating design is presented in Fig. 6-a. The experimental casting conditions were as follows: material ductile iron GCD600 which is similar to ISO600-3/S, green sand mold, cold box core, core and mold temperature $25^{\circ} \mathrm{C}$, pouring temperature $1420^{\circ} \mathrm{C}$. A schematic representation of measured shrinkage locations and frequency of defects on casting is displayed in Fig. 6-b.

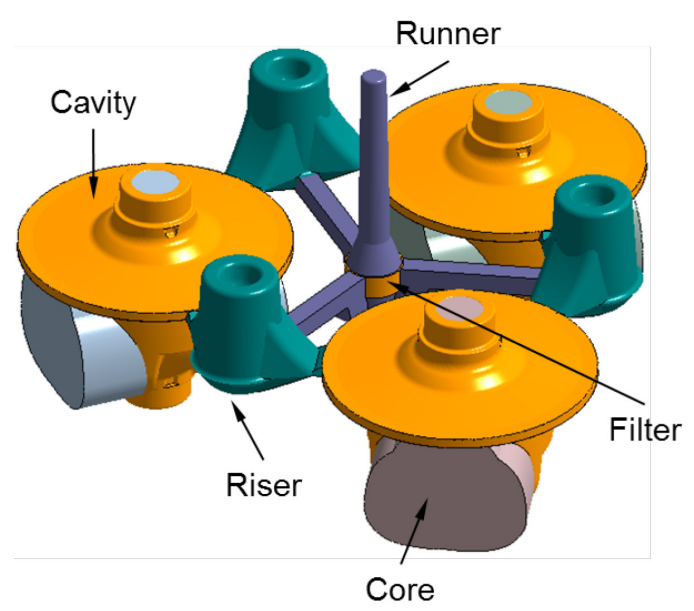

a) gating design including core, runner, riser and filter

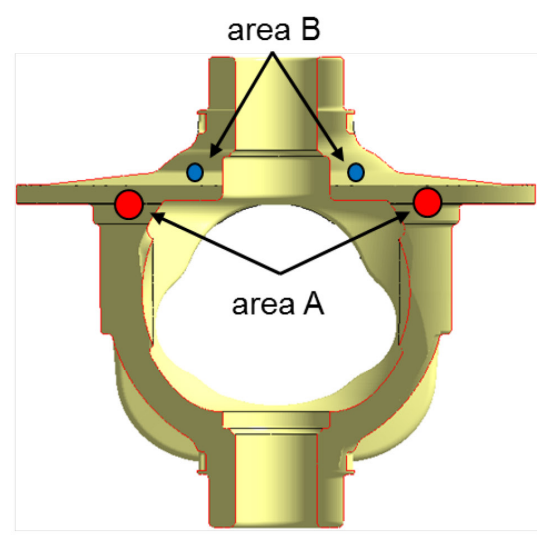

645 defects / 800 trials $(80.6 \%)$

164 defects / 800 trials $(20.5 \%)$

b) shrinkage defect of actual product in the foundry

Fig. 6. Gating design and shrinkage defects of a diffuser case made of ductile iron.

A comparison between the predictions of the RMS model combined with the probabilistic model at $\mathrm{CSF}=0.1[17]$ and those of the SIGAP model with the initial nitrogen content $\left[\mathrm{N}_{2}\right]=0.0001$ volume fraction [21] is displayed in Fig. 7. It is seen that the RMS (Fig. 7-a) predicts shrinkage defects of approximately the same size in the areas A and B in Fig. 6-b. However, the SIGAP model predicts higher gas porosity far higher in the area A than in B, as shown in Fig. 7-b. Thus, the SIGAP model has the advantage of quantitatively calculating the difference in the level of severity of shrinkage defects in castings; area B indicates 0.0022 volume fraction and A 0.005 volume fraction, which is 2.27 times more severe than B in shrinkage porosities.

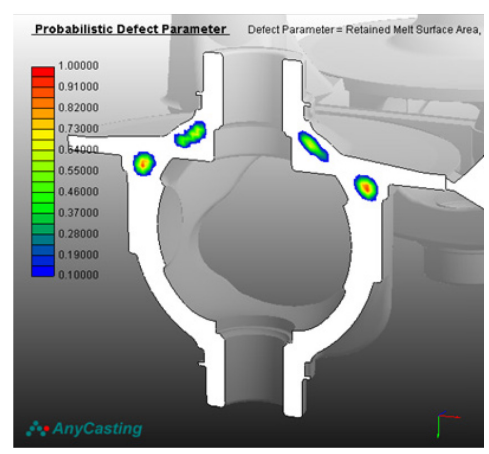

a) RMS (Retained Melt Surface) combined with the probabilistic model at $\mathrm{CSF}=0.1$

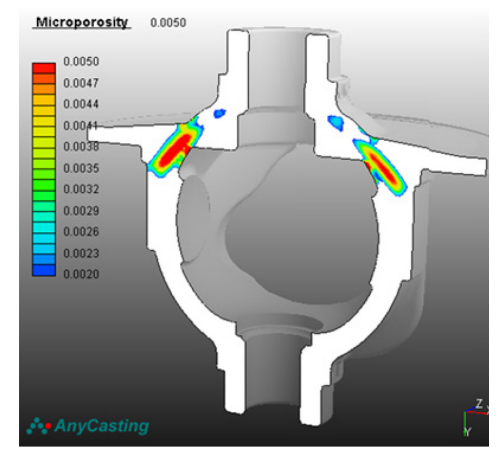

b) Gas amount calculated by SIGAP model with the initial $\left[\mathrm{N}_{2}\right]=0.0001$ volume fraction

Fig. 7. Comparison of shrinkage predictions with criterion-based and SIGAP models.

\section{Summary}

A numerical model for predicting shrinkage defects in iron castings based on gas pores formation during solidification is discussed. The model describes the evolution of gas concentration in the liquid, 
followed by gas pores nucleation and growth, and can predict shrinkage defects. After model calibration against literature data for microporosity, the model was successfully applied to the prediction of shrinkage defects in a ductile iron casting.

\section{Acknowledgements}

This work was supported by the Institute for Information \& Communications Technology Promotion (IITP) through a grant funded by the Korea government (MSIT) (No. 2016-0-00072, Development of Integrated Design Software and Optimization of Manufacturing Process in Automotive and Transport Equipment).

\section{References}

[1] T.S. Piwonka, M.C. Flemings: Trans. AIME, Vol. 236 (1966) pp. 1157-1165

[2] K. Kubo, R.D. Pehlke: Metallurgical Trans. B, Vol. 16B(2) (1985) pp. 359-366

[3] P.D. Lee, A. Chirazi, D. See: J. Light Metals, Vol. 1 (2001) pp. 15-30

[4] D.M. Stefanescu, Int. J. Cast Metals Res. Vol. 18(3) (2005) pp. 129-143

[5] E. Niyama, T. Uchida, M. Morikawa, S. Saito: AFS Cast Met. Res. J., Vol. 7 (1982) pp. 52-63

[6] A.S. Sabau and S. Viswanathan: Metall. Mater. Trans. B, Vol. 33B (2002) pp. 243-255

[7] P.K. Sung, D.R. Poirier, S.D. Felicelli: Modelling Simul. Mater. Sci. Eng., Vol. 10 (2002) pp. 551-568

[8] C. Pequet, M. Gremaud, M. Rappaz: Metall. Mater. Trans. A, Vol. 33A (2002) pp. 2095-2106

[9] A.V. Catalina, J.F. Leon-Torres, D.M. Stefanescu, M.L Johnson: in Solidification Processing 2007, ed. H. Johnes, Sheffield, The Univ. of Sheffield, (2007) pp. 699-703

[10] V. Khalajzadeh, K.D. Carlson, D.G. Backman, C. Beckermann, Metall. Mater. Trans A, 48A (2017), 1797-1816

[11] D.M. Stefanescu, A.V. Catalina: Int. J. Cast Metals Res. 24(3/4) (2011), 144-150

[12] E.F. Fisher, E.L. Roy, AFS Trans., 76 (1968), 237-290

[13] E.L. Roy, AFS Trans., 101 (1993), 961

[14] N. Roy, A.M. Samuel, F.H. Samuel: Met. Mater. Trans., 27A (1996), 415

[15] J. Campbell, in Modeling of Casting, Welding and Adv. Solidif. Proc. X, ed. D.M. Stefanescu et al., TMS, Warrendale, PA, (2003), 209-219

[16] A.V. Catalina, C.A. Monroe: in Modeling of Casting, Welding and Adv. Solidif. Proc. XIII, IOP Conf. Series: Materials Science and Engineering, 33 (2012) 012067

[17] S.B. Kim, Y.H. Yim, J.M. Yoon, S.H. Ahn, J.S. Baek, in: Proceedings of the 72nd World Foundry Congress, 21-25th May 2016, Nagoya, Japan

[18] S. Chang, D.M. Stefanescu: Acta. mater. 44(6) (1996), 2221-2235

[19] C.J. Vreeman et al.: Int. J. Heat \& Mass Transfer, 43 (2000), 677

[20] J.D. Zhu, S.L. Cockcroft, D.M. Maijer: Metall. Mater. Trans. A, 37 (2006), 1075-1085

[21] P.K. Sung, et al.: J. Crystal Growth, 226 (2001), 363-377 